\title{
Alcohol drinking rates of male between 7th and 11th graders in Japan decreased gradually based on nationwide repeated cross-sectional surveys from 1996 to 2008
}

\author{
Hideyuki Kanda ${ }^{1^{*}}$, Yoneatsu Osaki ${ }^{2}$, Yoshitaka Kaneita ${ }^{3}$, Osamu Itani ${ }^{4}$, Maki Ikeda ${ }^{4}$, \\ Takashi Ohida ${ }^{4}$, Susumu Higuchi ${ }^{5}$ \\ ${ }^{1}$ Department of Epidemiology and Public Health, School of Medicine, Yokohama City University, Yokohama, Japan; \\ *Corresponding Author: hkanda@yokohama-cu.ac.jp \\ ${ }^{2}$ Department of Environmental and Preventive Health, School of Medicine, Tottori University, Yonago, Japan \\ ${ }^{3}$ Department of Epidemiology and Public Health, School of Medicine, Ohita University, Ohita, Japan \\ ${ }^{4}$ Department of Public Health, School of Medicine, Nihon University, Tokyo, Japan \\ ${ }^{5}$ Kurihama Mecical Center, Yokosuka, Japan
}

Received 18 April 2013; revised 19 May 2013; accepted 6 June 2013

Copyright (C 2013 Hideyuki Kanda et al. This is an open access article distributed under the Creative Commons Attribution License, which permits unrestricted use, distribution, and reproduction in any medium, provided the original work is properly cited.

\begin{abstract}
Early drinking is considered to result in making tolerant of alcohol consumption and a higher prevalence of alcohol related disorders in the later. We focused generational impacts on drinking rate among high school students based on multiple nationwide data. The surveys were nationwide, cross-sectional random sampling surveys given every 4 years from the Japanese Youth Tobacco and Alcohol Surveys, 1996 to 2008. Participants were male 53,925 high school students from 7 th grade to 11th grade. We divided to the three follow-up groups every 4 years from 1996 to 2004 for male junior high school students in 7th grades and the end periods were 4 years later in 11th grades. Outcome measures in this study were life time drinking, current drinking within $\mathbf{3 0}$ days and weekly drinking. All drinking rates decreased each at 7 th and at 11th grade. The increments in these drinking rates from 7th grade to 11th grade in males decreased gradually in recent follow-up groups. Generational impacts should be considered by using follow-up groups to study drinking behaviors among students.
\end{abstract}

Keywords: Drinking Rates; Repeated

Cross-Sectional Study; National Wide Survey; Japan

\section{INTRODUCTION}

Alcohol drinking is one of the most important risk factor for lifestyle related diseases. Early drinking is considered to result in making tolerant of alcohol consumption and a higher prevalence of alcohol related disorders in the later [1].

Alcohol drinking rates among adolescents have also been reported [2-6]. However, most of these data were based on only cross-sectional studies. When estimating the rate increment for a follow-up group, the slope may be distorted by cohort effects [7-9]. The shapes of trends among adolescents by studying follow-up groups have not been examined at a national level. The variations in drinking rate among adolescents by follow-up groups have not been studied by follow-up groups at a national level, and no reported study has compared trends among multiple follow-up groups in a nation. Frequent observations are needed to compare trends in drinking rates among multiple follow-up groups belonging to the same grade in different years.

In this study, we looked at trends and generational impact on drinking rate by using follow-up groups of male Japanese high school students to quantitatively assess differences in trends. We used nationwide data from the Japanese Youth Tobacco and Drinking Surveys, gathered every 4 years between 1996 and 2008.

\section{SUBJECTS AND METHODS}

\subsection{Subjects}

The surveys were nationwide, cross-sectional random sampling surveys given every 4 years from 1996 to 2008, using the single-stage cluster sampling methodology [10]. 
Previous studies detailing the Japanese Youth Tobacco and Drinking Surveys have been reported [5,11-16]. The cluster unit of the sampling was schools. The survey targeted junior and senior high school students from schools selected through Japan using the National School Directory. All students enrolled in the sampled schools were subjects of the study.

The number of schools sampled in the 1996 survey was 122 of 11,274 junior high schools (selection rate: $1.1 \%)$ and 109 of 5501 senior high schools (2.0\%). Respective values for the 2000, 2004 and 2008 surveys were 132 of 11,200 junior (1.2\%) and 102 of 5315 senior high schools (1.9\%) in the 2000 survey, 131 of 11,060 junior (1.2\%) and 109 of 5272 senior high schools (1.9\%) in the 2004 survey, and 130 of 10,955 junior (1.2\%) and 110 of 5115 senior high schools (2.0\%) in the 2008 survey.

\subsection{Questionnaire}

We requested the cooperation of the principals of these schools and sent these individuals questionnaires for their respective school's student population. The teachers were asked to inform the students of the voluntary nature of their participation and to urge them to answer honestly. Anonymous questionnaires and envelopes were handed to the students for completion during school time. Upon completion, the questionnaires were sealed in the envelopes by the students themselves, collected by their teachers, and returned to our researchers unopened. This survey was reviewed and approved by the institutional review board of Nihon University (No. 19-5-0).

The questionnaire focused on drinking experience, drinking frequency and age. Experimenting drinkers, current drinkers, and weekly drinkers were defined as those who had tried drinking at least once, those who had drunk at least once in past 30 days, and those who had drunk at least once in past 7 days, respectively.

\subsection{Responses}

For the 1996 survey, responses were obtained from 80 junior (response rate $65.5 \%$ ) and 73 senior high schools (67.0\%). In 2000, the respective values were 99 (75.0\%) and 77 schools (75.5\%), and in 2004, there were 92 (70.2\%) and 87 schools (79.8\%). In 2008, the respective values were 92 (70.8\%) and 73 schools (72.7\%).

We used data from the 1996-2004 surveys for male junior high school students in 7 th grade, age 13 . We divided to the three follow-up groups every 4 years from 1996 to 2004. The end periods were 4 years later when they had become 11th graders, age 17, from 2000 to 2008. Table 1 shows a summary of follow-up groups. All group data includes all of the drinking rates from 7 th grade to 11th grade. The total sample size of the 1996-
Table 1. Follow-up groups of male students in this survey, Japan, 1996-2008.

\begin{tabular}{ccccc}
\hline \multirow{2}{*}{$\begin{array}{c}\text { Follow-up } \\
\text { groups }\end{array}$} & \multicolumn{2}{c}{ Years of Measurement } & \multicolumn{2}{c}{ Sample size } \\
\cline { 2 - 5 } & $\begin{array}{c}\text { Year in 7th } \\
\text { grade }\end{array}$ & $\begin{array}{c}\text { Year in } \\
\text { 11th grade }\end{array}$ & $\begin{array}{c}\text { Number } \\
\text { of 7th } \\
\text { grade }\end{array}$ & $\begin{array}{c}\text { Number } \\
\text { of 11th } \\
\text { grade }\end{array}$ \\
\hline 1996 group & 1996 & 2000 & 7,211 & 9,662 \\
2000 group & 2000 & 2004 & 8,248 & 12,241 \\
2004 group & 2004 & 2008 & 6,917 & 9,646 \\
\hline
\end{tabular}

2008 surveys was 53,925. We compared drinking rate increments from 7th to 11th grade for 3 follow-up groups as well as each drinking rate in both 7th grade and 11th grade for 3 follow-up groups.

\subsection{Statistical Analysis}

The percentages in the tables were calculated by a weighting method based on one-stage stratified cluster sampling [10]. We estimated the mean values of drinking rate by follow-up group. We calculated the change in drinking rate by subtracting the previous drinking rate from the current one, by follow-up group. Data were analyzed using the SPSS ver. 21.0 software (SPSS, Tokyo, Japan).

\section{RESULTS}

\subsection{Trends of Lifetime Drinking Rates}

Figure 1 shows the trends of the lifetime drinking rate for the three follow-up groups (1996, 2000 and 2004) of Japanese male students from 7 th grade to 11 th grade. The lifetime drinking rates decreased at 7 th grade in 2004 in males. They also decreased at 11th grade from 2000 to 2008. However, the increments in the lifetime drinking rate from 7 th grade to 11th grade in high school students were smaller in recent follow-up groups both in 2000 and in 2004 than in follow-up groups in 1996. Then, those increments in follow-up groups between 2000 and 2004 were similar; around $+15.6 \%-+15.9 \%$ in males from 7 th grade to 11 th grade.

\subsection{Trends of Current Drinking Rates}

Figure 2 shows the trends for current drinking rate for male students from 7th grade to 11th grade for three follow-up groups (1996, 2000, 2004). The current drinking rates decreased at 7th grade from 1996 to 2004 in males. They also decreased at 11th grade from 2000 to 2008. However, the current drinking rate increments from 7th grade to 11th grade decreased gradually one fourth from the 1996 follow-up group to the 2004 follow-up group. 


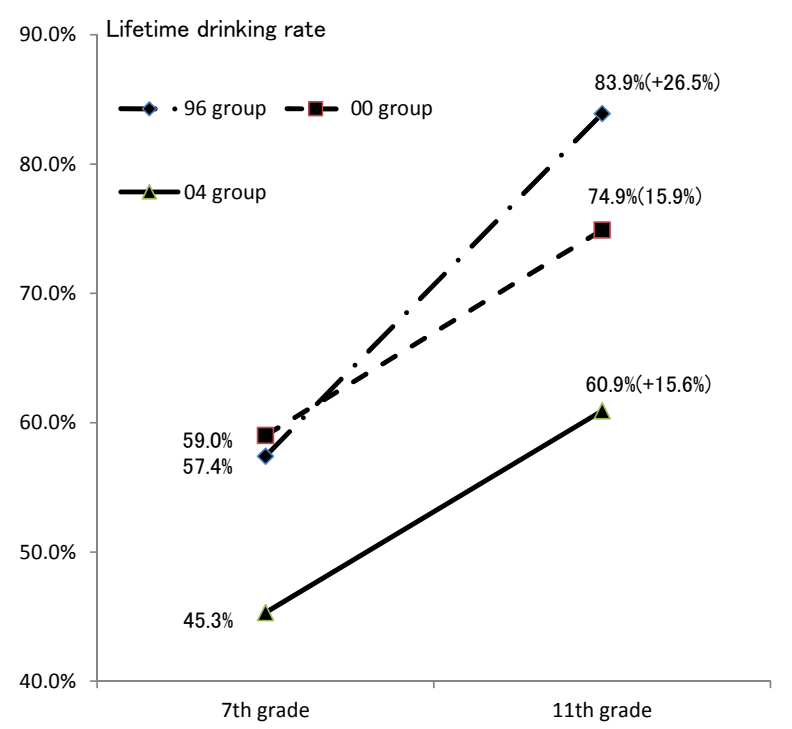

Figure 1. Lifetime drinking rate trends among male 7th - 11th graders by three follow-up groups (1996, 2000, 2004).

\subsection{Trends of Weekly Drinking Rates}

Figure 3 shows the trends for weekly drinking rate for male students from 7th grade to 11th grade for three follow-up groups (1996, 2000, 2004). The weekly drinking rates decreased at 7th grade from 1996 to 2004 both in males and females. They also decreased at 11th grade from 2000 to 2008. However, the weekly drinking rate increments from 7th grade to 11th grade grew gradually smaller from the 1996 follow-up group to the 2004 follow-up group; $+11.1 \%$ in the 1996 group, $+6.0 \%$ in the 2000 group and $+3.8 \%$ in the 2004 group.

\section{DISCUSSIONS}

Alcohol drinking rates for more recent male students' follow-up groups from 7th grade to 11th grade in Japan were relatively lower than older follow-up groups in 1996. Recent lifetime drinking rate increments from 7th grade to 11th grade were stable in the 2000 and 2004 follow-up groups, though all drinking rates decreased in both 7th and 11th grade from 1996 to 2004. Then, both current and weekly drinking rate increments from 7th grade to 11th grade grew gradually smaller from the 1996 follow-up group to the 2004 follow-up group. To our knowledge, this is the first report in the nationalwide survey to show the generational impact of drinking from 7th grade to 11th grade by follow-up groups. Our results show the importance of observing trends of drinking rates by using follow-up groups and monitoring a wide span of 4 years, not only at 7 th grade but also at 11th grade.

\subsection{Decreasing Factors of Drinking in Youth}

Although our study was restricted to the period

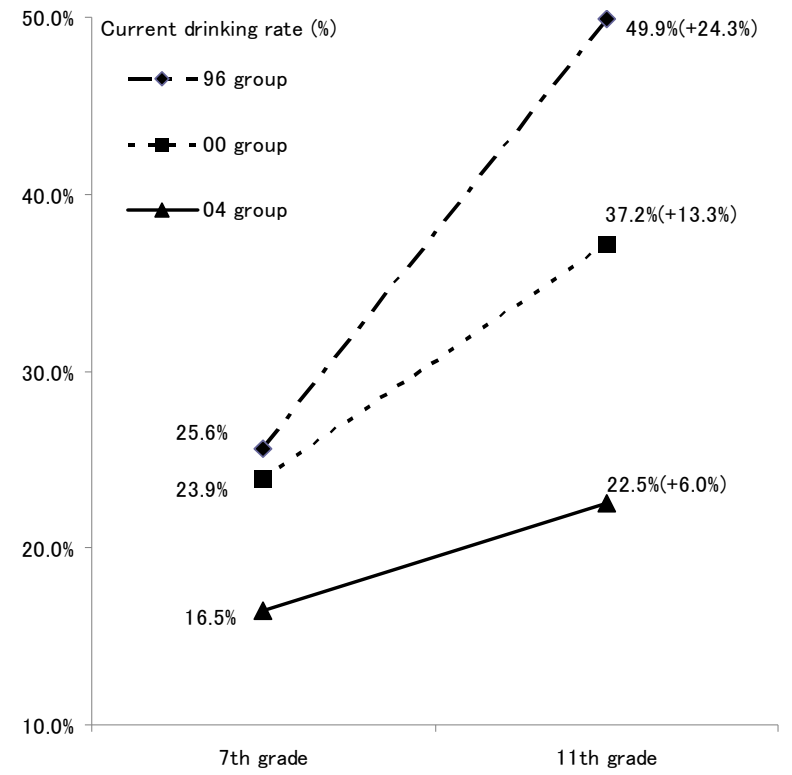

Figure 2. Current drinking rate trends among male 7th - 11th graders by three follow-up groups (1996, 2000, 2004).

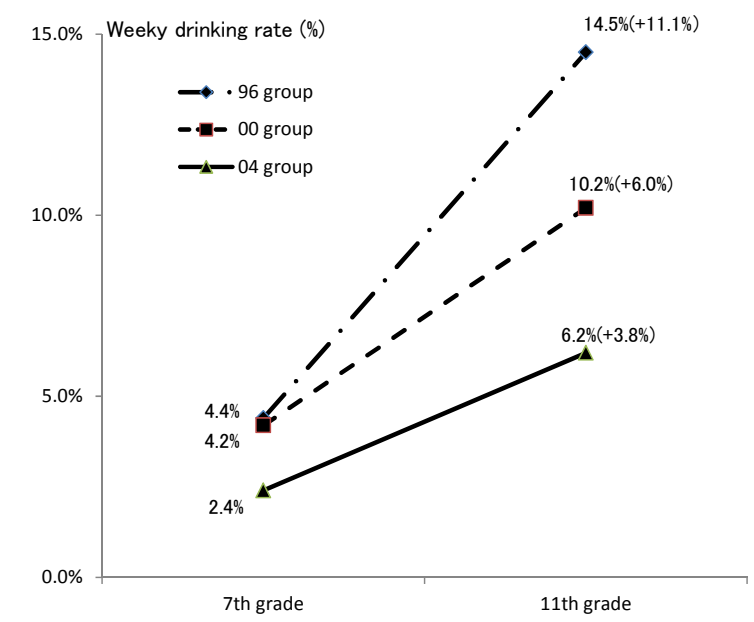

Figure 3. Weekly drinking rate trends among male 7th - 11th graders by three follow-up groups (1996, 2000, 2004).

from 7th grade to 11th grade among men, the drinking rate of the follow-up groups at 11th grade increased in each survey. Alcohol drinking rate is generally considered to increase with age. In recent follow-up groups, there has been a trend towards a decreased prevalence of drinking among adolescents in Japan.

There are several reasons to explain this result. First, alcohol policy may influence to youth. From 1997, warning label on the bottle has "prohibition of minor drinking” [17]. In 2000, Health People Japan, i.e., national plan of strategy for 21 century's health, was enacted [18]. This includes a goal of minor drinking to zero. Revision of Act Prohibit Minor from Alcohol Use and revision of liquor tax were also performed in 2000 [17]. By this re- 
vision of the act, age confirmation by retailers has become obligation and penalty has become severe. The National Tax Agency has got possible to cancel the license of liquor retailer which sold alcohol beverage to a minor, based on this revision of liquor tax [17]. The number of alcohol vending machines has decreased through voluntary regulation by industry from 2000 [19]. From 2003, severe punishment on drunk driving has started [20]. Additionally, voluntary regulation by a consultation of alcoholic beverage industry has begun on October 1st 2005, such as warning of prohibition of drinking before 20 years old on the TV commercials, avoiding the expression that a minor is interested in alcohol on the advertisements etc. [21]. However, many TV commercials for alcohol beverage are broadcasting still now. Second, parental drinking is one of the most important factors of adolescent drinking [22,23]. A decrease in the prevalence of drinking by adult men, including their fathers, probably contributed to the decrease among students. Peer drinking is also a well-known factor of adolescent drinking [24]. The decreased prevalence of drinking among students may also have contributed to a decrease in peer pressure to start drinking [24]. Finally, Japanese school students were given the opportunities to learn about alcohol and health in the life skill education in school from 2003. These alcohol policies and social environments contributed to the decrease of the drinking prevalence. Periodical nationwide surveys on adolescent drinking and survey of environmental factors are necessary to deal with the social problems of adolescent drinking.

\subsection{Alcohol Drinking Rates of Other Nations in Youth}

Considering the worldwide drinking epidemic rates in adolescents, prevention of drinking in the earlier stages of life is an important public health priority. In the United States, Finland and Sweden, drinking prevalence among adolescents decreased in the early 2000s. However, the majority of the world has not observed any decrease in drinking prevalence. There are global trends to restrict drinking among adolescents based on the WHO global strategy to reduce the harmful use of alcohol [25]. Some countries may have begun taking action quickly to ensure implementation of this strategy. Our study focused on generational impacts on drinking by looking at follow-up groups. Few studies clarified these impacts in the world. In the future, the effects of generational impact need to be considered when monitoring follow-up groups for drinking prevalence.

\subsection{Repeated Cross-Sectional Study}

A specific feature of this study was the use of a nationwide, repeated, cross-sectional survey with random sampling to show the slope by follow-up group. In a cross-sectional study, each student at 7th grade is observed once, and in a longitudinal study each student is observed two times. In a repeated, cross-sectional survey, each student is observed once, but each follow-up group is studied two times. Thus, we can infer the slope of a follow-up group from a repeated, cross-sectional survey. A repeated, cross-sectional survey is usually reported as a cross-sectional curve by survey year [26], but such a cross-sectional slope is different from the slope of a follow-up group when a follow-up group effect occurs. In a cross-sectional slope, we see combined effects of follow-up group and year. When interpreting drinking rates twice, as we did here, the follow-up group effect should be considered. A repeated, regular survey is suited to monitoring trends by follow-up group because trends can be produced and checked for gradual changes by generation. The weakness of a repeated, cross-sectional survey, compared with a longitudinal study, is that all inferences are described in terms of population averages, and the variability of trends among individuals and the effects of covariates cannot be inferred. The slopes of the drinking rate obtained by following a single person longitudinally and that obtained by population average are well known to be different because the timing of drinking initiation varies greatly between individual people. A repeated, cross-sectional survey and a longitudinal study should be considered as being complementary.

\subsection{Limitations}

One limitation of this study is the possibility of misclassification of drinking status among students. Although this study uses an anonymous questionnaire survey, the respondents may have been reluctant to report their actual drinking status due to more active antidrinking policies in recent years. However, we considered the influence of misclassification of reported drinking status to not be large because the number of questionnaires with invalid or contradictory answers did not increase during this study period. Then, we focused only men at 7th graders in the baseline. Confirmation of the persistence of this decrease in prevalence will require periodic monitoring of adolescent drinking prevalence and related factors.

\subsection{Conclusions}

Alcohol drinking rates among Japanese male students decreased both at 7th grade and at 11th grade. However, the drinking rate increments from 7th grade to 11th grade were smaller in recent follow-up groups than in older follow-up groups, which means most Japanese high school students did not start drinking cigarettes in the early 2000s. We have shown that monitoring trends by 
follow-up group are important in studying drinking for public health. Values of drinking rates from a young age to adolescence by follow-up group should be examined. For this purpose, a repeated, cross-sectional survey is suitable. Values of drinking rate and regular changes in drinking rate are important because drinking rate changes dynamically during adolescence. Not only trends in drinking rates at fixed ages, but also generational impacts should be considered by using follow-up groups to study drinking behaviors among students. In the future, we need to continue careful monitoring of follow-up group drinking prevalence. When long-term health promotions are planned or assessed, policy makers need to look at changes in follow-up groups.

\section{ACKNOWLEDGEMENTS}

We are grateful to Mr. Michita Nagastuka, Ms. Sanae Numaguchi and Ms. Kyoko Kawamoto for the assembling of the data, and to Ms. Jenifer Shunk Monma for the English editing of the manuscript. This study was supported by a grant for a Special Research Project in 1996 and a Public Health Research Projects in 2000, 2004 and 2008 from the Ministry of Health and Welfare Health Science Research Fund in Japan. The sponsors of this study had no role in study design, data collection, data analysis, data interpretation and writing of the paper.

\section{REFERENCES}

[1] Hingson, R.W., Heeren, T. and Winter, M.R. (2006) Age at drinking onset and alcohol dependence: Age at onset, duration, and severity. Archives of Pediatrics \& Adolescent Medicine, 160, 739-746.

doi:10.1001/archpedi.160.7.739

[2] Toumbourou, J.W., Hemphill, S.A., McMorris, B.J., Catalano, R.F. and Patton, G.C. (2009) Alcohol use and related harms in school students in the USA and Australia. Health Promotion International, 24, 373-382. doi:10.1093/heapro/dap037

[3] Kann, L., Kinchen, S.A., Williams, B.I., Ross, J.G., Lowry, R., Grunbaum, J.A., et al. (2000) Youth risk behavior surveillance-United States, 1999. MMWR CDC Surveillance Summaries, 49, 1-32.

[4] Chou, P., Liou, M.Y., Lai, M.Y., Hsiao, M.L. and Chang, H.J. (1999) Time trend of substance use among adolescent students in Taiwan, 1991-1996. Journal of the Formosan Medical Association, 98, 827-831.

[5] Suzuki, K., Osaki, Y., Wada, K., Matsushita, S., Hayashi, K., Ohida, T., et al. (2007) Decreases in Japanese adolescent drinking in 1996, 2000 and 2004 national surveys of Japanese junior and senior high school students. Japanese Journal of Alcoholism and Drug Dependence, 42, 138-151.

[6] Currie, C., Roberts, C., Morgan, A., Smith, R., Settertobubulte, W., Samdal, O., et al. (2004) Young people's health in contexts. Health Behavior in School-aged Children (HBSC) study: International report from the 2001/2002 survey. WHO regional office for Europe, Copenhagen.
[7] Szklo, M. and Nieto. F.J. (2000) Epidemiology: Beyond the basics. Aspen Publishers, Frederick.

[8] Twisk, J.W.R. (2004) Applied longitudinal data analysis for epidemiology: A practical guide. Cambridge University Press, Cambridge.

[9] Fitzmaurice, G.M., Laird, N.M. and Ware J.H. (2004) Applied longitudinal analysis. John Wiley \& Sons, Hoboken.

[10] Cochran, W.G. (1977) Single-stage cluster sampling: Clusters of unequal sizes, in: Sampling techniques. Wiley, New York.

[11] Suzuki, K., Minowa, M., Osaki, Y. and Wada, K. (2001) Drinking behaviors of Japanese adolescents' problem drinker-Report of 1996 national survey. Japanese Journal of Alcoholism and Drug Dependence, 36, 39-52.

[12] Osaki, Y., Minowa, M., Suzuki, K. and Wada, K. (1999) Nationwide survey on alcohol use among junior and senior high school students in Japan. Japanese Journal of Alcoholism and Drug Dependence, 46, 883-893.

[13] Suzuki, K., Minowa, M. and Osaki, Y. (2000) Japanese national survey of adolescent drinking behavior in 1996. Alcoholism: Clinical and Experimental Research, 24, 377-381. doi:10.1111/j.1530-0277.2000.tb04626.X

[14] Osaki, Y., Tanihata, T., Ohida, T., Kanda, H., Suzuki, K., Higuchi, S., et al. (2009) Decrease in the prevalence of adolescent alcohol use and its possible causes in Japan: Periodical nationwide cross-sectional surveys. Alcoholism: Clinical and Experimental Research, 33, 247-254.

[15] Osaki, Y., Minowa, M., Suzuki, K. and Wada, K. (2003) Adolescent alcohol use in Japan, 1996. Yonago Acta Medica, 46, 35-43.

[16] Suzuki, K., Osaki, Y., Minowa, M., Wada, K., Ohida, T., Doi, Y., et al. (2003) Japanese national survey of adolescent drinking behavior: Comparison between 1996 and 2000 surveys. Japanese Journal of Alcoholism and Drug Dependence, 38, 425-433.

[17] Fukawa, Y. (2011) Atlas alcohol tax. Japanese National Finance Association, Tokyo.

[18] Shimizu, S., Kim, D.S. and Hirota, M. (2004) Drinking practice and alcohol-related problems: The national representative sample survey for Healthy Japan 21. Japanese Journal of Alcoholism and Drug Dependence, 39, 189206.

[19] Higuchi, S., Matsushita, S., Maesato, H. and Osaki, Y. (2007) Japan: Alcohol today. Addiction, 102, 1849-62. doi:10.1111/j.1360-0443.2007.01902.x

[20] Nagata, T., Setoguchi, S., Hemenway, D. and Perry, M.J. (2008) Effectiveness of a law to reduce alcohol-impaired driving in Japan. Injury Prevention, 14, 19-23. doi:10.1136/ip.2007.015719

[21] Yoshida, S., Sakamaki, S. and Tanioka, T. (2011) Relation between television commercial of alcoholic beverage and alcoholism. Japanese Journal of Alcoholism and Drug Dependence, 46, 560-569.

[22] Latendresse, S.J., Rose, R.J., Viken, R.J., Pulkkinen, L., Kaprio, J. and Dick, D.M. (2008) Parenting mechanisms in links between parents' and adolescents' alcohol use behaviors. Alcoholism: Clinical and Experimental Research, 
32, 322-330. doi:10.1111/j.1530-0277.2007.00583.x

[23] Seljamo, S., Aromaa, M., Koivusilta, L., Rautava, P., Sourander, A., Helenius, H., et al. (2006) Alcohol use in families: A 15-year prospective follow-up study. Addiction, 101, 984-992. doi:10.1111/j.1360-0443.2006.01443.x

[24] Johansen, A., Rasmussen, S. and Madsen, M. (2006) Health behaviour among adolescents in Denmark: Influence of school class and individual risk factors. Scandinavian Journal of Public Health, 34, 32-40. doi:10.1080/14034940510032158

[25] Chick, J. (2011) The WHO global strategy to reduce the harmful use of alcohol. Alcohol and Alcoholism, 46, 223. doi:10.1093/alcalc/agr035

[26] Kiriike, N., Nagata, T., Sirata, K. and Yamamoto, N. (1998) Are young women in Japan at high risk for eating disorders? Decreased BMI in young females from 1960 to 1995. Psychiatry and Clinical Neurosciences, 52, 279281. doi:10.1046/j.1440-1819.1998.00387.x 